\title{
Automatic vigilance for task-related information: Perseverance after failure and inhibition after success
}

\author{
KLAUS ROTHERMUND \\ University of Trier, Trier, Germany
}

\begin{abstract}
In three experiments, the influence of failure and success on persistent automatic vigilance for taskrelated information was investigated. Participants first had to work on a series of synonym selection problems for which negative and positive feedback was given independently of their performance. In the second part of the experiments, words from the synonym selection problems were presented as distractors in a dual task with speeded responses. Interference effects of the distractors served as a measure of automatic vigilance for the previous synonym selection problems that was unbiased by strategic processes. Interference effects were stronger for words from failure tasks than for words from success tasks. Comparing the success and failure conditions against a neutral baseline suggested that this difference was due to both a perseverance of automatic vigilance for failure tasks and an inhibition of cognitive accessibility after success.
\end{abstract}

Automatic attention allocation can be seen as an interface that mediates the influences of motivational states on cognitive processing (Derryberry \& Tucker, 1994; Rothermund, Wentura, \& Bak, 2001). Adopting a goal or a task is accompanied by a corresponding attentional set that biases automatic cognitive processing in favor of goal- or task-related information (Houghton \& Tipper, 1994; Pashler, 1998). The cognitive accessibility of information relating to a current goal or action plan is increased, and goal-related stimuli automatically attract attention (Klinger, 1996; Moskowitz, 2002; Riemann \& McNally, 1995). This automatic vigilance mechanism reflects a relevance principle of information processing that guarantees a chronically increased sensitivity for information relating to a current goal or task.

The aim of the present article is to investigate the fate of goal-related attentional sets when goal pursuit has failed. It is typically assumed that automatic vigilance for goal-related information is terminated after the goal has been attained (Anderson, 1983). But what happens to a goal- or task-related attentional set when the goal or task objective turns out to be unattainable? Assume that you have finally dared to invite the person you adore to the cinema but that your offer has been rejected, implying that he or she is already attached to someone else. Will the cognitive set that you have already developed for this person and that has drawn your attention and thoughts toward him or her during the past weeks persist, or will it decay?

From an action-theoretical perspective, it is important not to equate current goals that are still attainable with

Correspondence concerning this article should be addressed to K. Rothermund, Department of Psychology, University of Trier, D-54286 Trier, Germany (e-mail: rothermu@uni-trier.de). goal intentions that have already failed. In the case of a goal intention or task that is currently pursued or assumed to become relevant in the near future, an automatic vigilance mechanism increases the sensitivity for information that is relevant for that task (Goschke \& Kuhl, 1993, 1996; Klinger, 1996). Maintaining a goalrelated attentional set after failure, however, is no longer adaptive, because information relating to an unattainable goal is no longer relevant for the regulation of behavior (Brandtstädter \& Renner, 1992; Brandtstädter \& Rothermund, 2002a, 2002b). Instead, such a perseveration of an increased sensitivity for information relating to an unattainable goal might foster chronic mental rumination (Kuhl \& Helle, 1986; Martin \& Tesser, 1989) and might also delay psychological adjustment after failure by preventing such processes as reorientation or disengagement from barren commitments (Brandtstädter \& Renner, 1992; Brandtstädter \& Rothermund, 2002a, 2002b; Klinger, 1975). Despite these dysfunctional side effects, a perseveration of automatic vigilance after failure might nevertheless constitute a fairly general phenomenon that simply reflects the other side of the coin of the goal vigilance mechanism.

Lewin $(1926,1935)$ was the first to propose that an attentional set remains active until the corresponding goal is achieved. This perseverance hypothesis entails that attentional control settings are maintained even in the face of failure. Accordingly, information relating to a blocked goal or task should remain in a state of heightened accessibility. The first empirical studies in which the perseverance hypothesis was investigated were conducted by Zeigarnik (1927; see Lewin, 1935, pp. 243-245, for a short English summary of Zeigarnik's experiments). First, Zeigarnik's participants had to perform a number 
of different tasks, half of which were terminated by the experimenter before completion, thus rendering the goal of completing the tasks definitely unattainable. In the second part of the experiments, the participants were unobtrusively asked to recall the previous tasks. The main finding was that incomplete tasks were recalled better than completed tasks. Marrow (1938) demonstrated that it is the experience of failing to reach a goal standard that is associated with increased recall, and not the interruption or incompleteness of a task per se: Recall was better for noninterrupted tasks when participants were told that a task was terminated as soon as the experimenter was satisfied with their performance-that is, when noninterruption indicated failure to reach a given performance standard.

Subsequent research with the Zeigarnik paradigm revealed, however, that enhanced recall of unfinished tasks is not a robust effect (Butterfield, 1964; van Bergen, 1968). In some studies, even an "inverse" Zeigarnik effect was found - that is, recall was better for successfully completed tasks (Holmes, 1990). This instability of the Zeigarnik effect was sometimes attributed to the influence of self-presentational motives on memory search and recall: Participants might want to create a favorable impression of themselves by trying to recall successful tasks. This explanation was supported by the finding that a reversed Zeigarnik effect was observed mainly under conditions of high task importance and when there was a high involvement of self-esteem or other self-related motives (Greenwald, 1982; Holmes, 1990). Accordingly, a neutralization or reversal of the Zeigarnik effect could reflect a motive-driven search for or report of successfully completed tasks, rather than a reduced automatic vigilance for information relating to failure tasks.

Thus, a major interpretational problem of previous findings with the Zeigarnik paradigm relates to the dependent variable that was used: The free recall test cannot be taken as a direct and unbiased measure of automatic vigilance or cognitive accessibility, because recall frequencies can be influenced by a strategic memory search or by response tendencies. Up to now, only a few experiments have been reported that have tried to measure the cognitive accessibility of failure- and success-related information more indirectly. In one experiment, perceptual thresholds for words relating to previous success and failure tasks were estimated (Postman \& Solomon, 1949). Martin, Tesser, and McIntosh (1993, Experiment 3) used a task in which participants had to guess the identity of words that were presented one letter at a time and that were related or unrelated to an unattained goal. Beckmann (1996, Experiment 2) used recognition latencies to measure the cognitive activation of information relating to previous success and failure tasks. The perseverance hypothesis was supported in the studies of Beckmann (1996) and Martin et al. (1993) — that is, recognition latencies were faster and guessing rates were higher for failure-related words. Postman and Solomon (1949), however, found no effect of previous success or failure feedback on perceptual thresholds for task-related words.
Although the dependent variables used in these more indirect studies are less sensitive to strategic factors, it can be questioned whether these measures are completely immune to influences of a biased memory search or report strategies. In the perceptual threshold and guessing tasks, for example, participants might sooner or later become aware of the fact that some of the target stimuli relate to the previous success and failure episodes; in the case of the recognition task, this relation is an essential feature of the task itself. Noticing this relation might invite participants to generate hypotheses for the targets of the following trials by actively retrieving the previous episodes. Therefore, performance in these tasks can again be influenced by strategic processes-that is, by a selective retrieval of success (or failure) episodes or by a selective responding to success (or failure) targetsand might not reflect true levels of automatic cognitive accessibility.

Such a contamination with the effects of strategic processing was demonstrated for different indirect or implicit measures of memory that employ a facilitation logic (Jacoby, 1991; Russo \& Parkin, 1993). The critical feature of facilitation measures is that performance on these measures will benefit from a strategic recall of previous episodes. Taking into consideration this confounding of automatic and strategic processes might also explain why the studies just mentioned still yielded inconsistent results. For example, the effects of strategic response generation and response suppression can have a marked effect in a perceptual threshold task when the effects of response bias are not controlled (Eriksen, 1958). These effects might have counteracted accessibility effects in the study by Postman and Solomon (1949), whereas it might have had less of an effect in the tasks that were used by Beckmann (1996) and Martin et al. (1993).

The perseverance hypothesis is thus still in need of a more stringent test. Such a test should satisfy the requirement that the criterion variable is definitely immune to the influence of strategic processes (biased memory search or report). The research presented in this article provides such a test. In the present experiments, stimuli relating to previous success and failure tasks were presented as distractor stimuli in a subsequent reaction time task. Interference effects of the distractor stimuli in this task were used to measure the persistence of automatic vigilance for the previous success or failure tasks. By analyzing interference effects, an interpretation in terms of strategically biased information processing or response strategies can be ruled out for the following reasons (cf. Jacoby, 1991).

1. Strategic processing of the distractors runs counter to the explicit instructions of the reaction time task, which requires that participants ignore the irrelevant stimuli.

2. Processing of the distractors is incompatible with efficient processing of the relevant information contained in the target stimuli of the reaction time task. To achieve the best performance in the reaction time task, participants will attempt to ignore the distractors. Inter- 
ference effects caused by task-irrelevant distractors thus fulfill the strictest criterion of automaticity, because they occur despite an intention to ignore the distractors (Bargh, 1989).

3. Distractors are irrelevant to the reaction time task and do not require a response, which rules out explanations in terms of response tendencies.

4. As an additional advantage, the distractors will barely be noticed by the participants, which makes explanations in terms of processing or response strategies unlikely.

\section{EXPERIMENT 1}

The experiment consisted of two parts. In the first part, the participants had to perform a series of synonym selection problems. The objective of these tasks was to find the closest synonym (the solution word) to a given target word out of a number of response alternatives (e.g., frugal [target]-huge, tasty, sparing [solution], parsimonious, rustic). Failure or success was induced by giving either negative or positive feedback after each of these problems. Feedback was manipulated independently of whether the correct solution was selected. Using difficult synonym selection problems prevented the participants from recognizing that feedback was not veridical, because they could not be sure whether their answer had been correct.

In the second part of the experiment, automatic vigilance for information relating to the previous synonym problems was measured. For this purpose, the target words from the synonym problems were presented as irrelevant distractor stimuli in a combined naming and detection task. An automatic vigilance mechanism for information relating to unfinished or incomplete goals should produce attentional capture for the distractor stimuli relating to the previous synonym selection problems that had received a negative feedback. To obtain a highly sensitive measure of distractor interference, the naming task was combined with a secondary tone detection task (cf. Bargh, 1982; McCabe \& Gotlib, 1993; Posner \& Boies, 1971). Attentional capture by the distractor stimuli should be most critical for performance in the secondary tone detection task, for the following reason: In a dual-task situation, response selection in the secondary task has to be postponed until response selection in the primary task has been completed (e.g., Pashler, 1998).

The process of switching between mental sets relating to different tasks, however, requires executive control processes and is extremely dependent on attentional resources (e.g., task-shifting costs are inversely related to working memory capacity and other indicators of executive functioning; Engle, 2002; Mayr \& Kliegl, 2000; Rubinstein, Meyer, \& Evans, 2001). Switching between primary and secondary tasks is thus the most vulnerable point for interference effects of task-unrelated distractors. An intrusion of the distractors into the resourcedemanding process of switching between two tasks should affect response times primarily in the task that is executed second. The mere execution of an already prepared response to the first task should not be affected much by distractor interference. In conclusion, automatic allocation of attention to distractor stimuli should delay responding primarily in the tone detection task. The perseverance hypothesis predicts that interference effects will be stronger for distractor words relating to synonym problems for which a negative feedback had been given during the first part of the experiment.

\section{Method}

Participants. Thirty-two University of Trier undergraduates (16 women and 16 men) volunteered in exchange for partial credit toward course requirements. The experiment was conducted in individual sessions of approximately 30-min duration.

Materials. A pilot study $(n=16)$ was conducted to select the synonym problems for the experiment: 40 synonym selection problems taken from the Similar Word Meaning subtest of a German intelligence test (Jäger \& Althoff, 1983) were presented on a computer screen. Each problem consisted of a target stimulus and five response alternatives containing the correct solution. The participants had to select the response stimulus for which the meaning was closest to the meaning of the target word. After that, the participants rated their confidence in their decision on a 7-point scale. A total of 15 synonym problems was selected for the experiment; eight difficult problems were used as manipulated feedback problems ( $e x$ perimental problems; correct responses in the pilot study, $45 \%$; mean confidence, 4.55). These 8 problems were split into two sets of 4 problems each. Each participant received positive feedback for one set of problems, whereas negative feedback was given for the other set of problems. Assignment of sets to the feedback conditions was counterbalanced across participants. The two sets were comparable with respect to difficulty, time to solution, and length of the target words. Seven additional problems were selected, with six presented as introductory problems and one as the final problem during the experiment.

For the combined naming and tone detection task, 90 neutral word pairs were generated for 90 trials of the naming task, one word being the target and one word being the distractor stimulus of the respective trial. The first 10 trials were used as practice trials (a tone was presented in 2 of these trials). Of the remaining 80 trials, 16 trials were selected for tone presentation. In 8 of these trials, the distractor words were the target words of the eight experimental synonym problems. The remaining 8 trials with a tone served as filler trials to prevent a detection of a covariation between the content of the distractor stimuli and the appearance of the tone.

Design. Feedback (negative or positive) was manipulated within subjects for the experimental problems. Assignment of the two sets of experimental problems to the feedback conditions was counterbalanced across participants according to a Latin square. The order in which positive and negative feedback was given for the experimental synonym problems was counterbalanced across participants to prevent a confounding of feedback with position and sequence effects.

Procedure. The participants were told that they would work on different subtests of a newly developed computer version of an intelligence test. For each synonym problem, a response had to be given within $20 \mathrm{sec}$, with the last $5 \mathrm{sec}$ marked by short beeps. Immediately after the registration of a response, the stimuli of the synonym problem were deleted from the screen, and a positive or negative feedback sentence was shown for $2 \mathrm{sec}$ in the middle of the screen (You did/did not find the optimal solution). Feedback was veridical for the introductory and final tasks. For the experimental tasks, feedback was given according to the predetermined feedback schedule (see the Design section). 
Immediately after the feedback for each synonym problem, a distractor task was presented. A random three-digit number was presented on the screen, and the participants had to count backward in steps of seven for $10 \mathrm{sec}$. The next synonym problem was presented immediately afterward. The distractor task prevented rumination over the synonym problem and ruled out a differential encoding of the problems on the basis of the feedback (Caron \& Wallach, 1957).

The combined naming and tone detection task was presented after the synonym problems. The first 10 trials were given as practice trials. After that, the remaining 80 trials, which also contained the target words of the experimental synonym problems as distractor stimuli, were presented in an individually randomized sequence (words of the response set of the synonym problems were not used as distractors, to prevent any confound with response selection by the participants). Throughout the task, a rectangular white frame was shown in the middle of a VGA screen on an otherwise black background. Each trial consisted of the following sequence (see Figure 1). Three words appeared in the frame. The middle word (target) was to be named aloud as quickly as possible. The distractor words were presented directly above and below the target word. The stimuli remained on the screen for $750 \mathrm{msec}$ and were then replaced by a mask containing three rows of small schema faces. The mask remained on the screen for 1,250 msec. Afterward, the frame was cleared, and the stimuli of the next trial were presented after an intertrial interval of $1,000 \mathrm{msec}$. In the trials containing a tone, $300 \mathrm{msec}$ after the onset of the word stimuli, a $440-\mathrm{Hz}$ tone signal was presented for $50 \mathrm{msec}$ via headphones. In these trials, in addition to naming the target word, the participants had to press a key on the computer keyboard as quickly as possible. Tone detection latencies were recorded to the nearest millisecond. Naming latencies were registered by a voice key apparatus realized by means of a microphone connected to a SoundBlaster audio card.

Following the combined naming and tone detection task, the participants were probed for suspicion. As part of a larger questionnaire, the participants were asked if they had noticed anything un- usual while working at the tasks. The participants were then asked explicitly whether they had had doubts about the validity of the feedback in the synonym problems and whether they had recognized that some of the words from the synonym problems had been presented during the naming task. No participant was suspicious about the validity of the feedback or reported noticing the appearance of synonym problem words in the naming task. After this interrogation, the participants were thanked and debriefed.

\section{Results}

Tone detection latencies. Reaction time in the secondary tone detection task was the primary dependent variable in the present experiment. Trials in which no response was registered until the beginning of the next trial (1.0\% of all the trials) as well as reaction times that could be considered outlier values ${ }^{1}$ ( $2.9 \%$ of all the responses) were excluded from further analyses. For each participant, mean reaction times were computed separately for trials containing distractor stimuli referring to synonym problems with positive and negative feedback. Mean response latencies in the tone detection task are shown in Table 1. On average, reaction times were $34 \mathrm{msec}$ longer for the trials that contained distractor stimuli referring to negative feedback problems than for the trials with positive feedback distractors. This difference was significant, by subjects $[t(31)=4.66, p<.001 ; d=.82]$ and by items $[t(7)=4.96, p<.001 ; d=1.75]$.

Naming latencies. An analogous analysis of the naming latencies revealed a similar but weaker pattern of results. On average, naming latencies were $16 \mathrm{msec}$ longer for the trials containing distractor words from the nega-

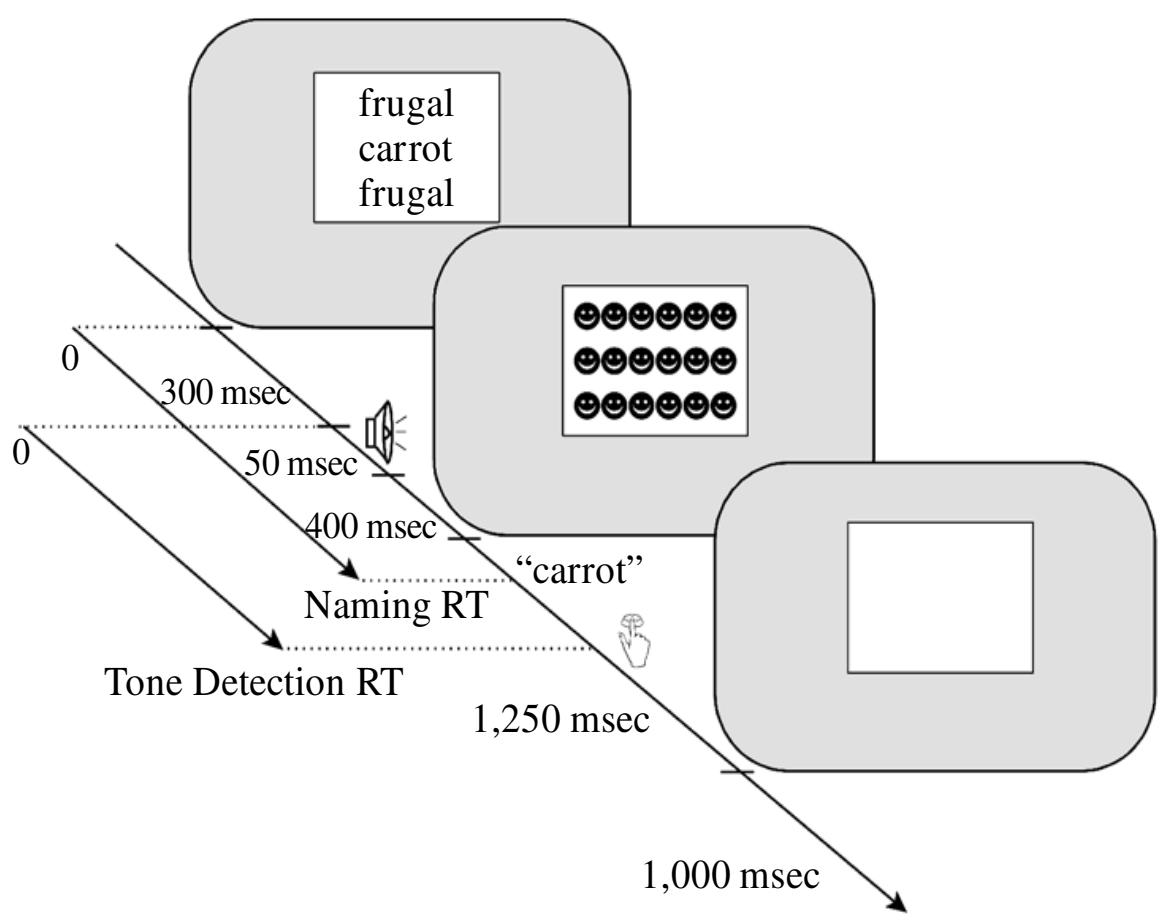

Figure 1. Schematic illustration of a trial sequence in Experiment 1. See the text for further explanations. 
Table 1

Tone Detection Latencies (in Milliseconds; Experiments 1 and 2) and Grammatical Categorization Latencies (Experiment 3) for Trials With Different Types of Distractor Words

\begin{tabular}{|c|c|c|c|c|c|c|c|c|}
\hline \multirow[b]{3}{*}{ Task } & \multicolumn{8}{|c|}{ Distractor Type } \\
\hline & \multicolumn{2}{|c|}{ Success } & \multicolumn{2}{|c|}{ Failure } & \multicolumn{2}{|c|}{ No Feedback } & \multicolumn{2}{|c|}{ Not Presented } \\
\hline & $M$ & $S E$ & $M$ & $S E$ & $M$ & $S E$ & $M$ & $S E$ \\
\hline \multicolumn{9}{|l|}{ Tone detection } \\
\hline Experiment 1 & 391 & 18 & 425 & 19 & - & - & - & - \\
\hline Experiment 2 & 351 & 11 & 371 & 10 & 360 & 10 & 359 & 11 \\
\hline \multicolumn{9}{|c|}{ Grammatical categorization } \\
\hline Experiment 3 & 1,173 & 45 & 1,279 & 53 & 1,238 & 49 & - & - \\
\hline
\end{tabular}

tive feedback problems $(M=624 \mathrm{msec})$ than for the trials with distractors from the positive feedback tasks $(M=609 \mathrm{msec})$ by subjects $[t(31)=1.70, p<.10 ; d=$ $.30]$ and by items $[t(7)=2.70, p<.05 ; d=.95]$.

\section{Discussion}

The results supported the perseverance hypothesis. The target words of the synonym problems for which negative feedback had been given produced increased interference effects in a subsequent reaction time task. The observed effect was much more pronounced for the secondary tone detection task than for the naming task. As was argued above, this difference might be due to the fact that distractor interference primarily affects the process of switching between mental sets referring to the two tasks and should, thus, delay responding primarily in the secondary task. ${ }^{2}$

Importantly, the interference effects of the distractor stimuli in the reaction time task cannot be explained by means of strategic processes (biased search or responding). An explanation of the findings in terms of a differential encoding of success and failure problems is also unlikely, because a cognitively demanding distractor task was presented after each synonym selection problem immediately after success or failure feedback had been given. Working on the distractor tasks prevented a further processing of the preceding synonym selection problems. A differential encoding of the synonym tasks on the basis of the feedback can, therefore, be ruled out. The observed effect can thus be attributed to differences in the persistence of automatic vigilance for success and failure tasks. This finding is in line with the results of recent studies by Beckmann (1996) and Martin et al. (1993) that also used indirect measures to estimate automatic vigilance for failure- versus success-related information.

Although the present experiment avoids interpretational problems with regard to the nature of the underlying processes, another important argument can be raised against an interpretation of the effect in terms of a perseverance of vigilance after failure. A perseverance interpretation presupposes that the success condition provides an adequate neutral baseline against which effects of failure can be tested. It is assumed that the successful execution of a task leads to the deactivation of a previous attentional set, which reduces the activation of task-related in- formation to a neutral baseline level (Anderson, 1983; Klinger, 1975). This assumption, however, can be questioned. Recent experiments by Marsh and colleagues suggest that task-related information is actively inhibited after the successful execution of a task (Marsh, Hicks, \& Bink, 1998; Marsh, Hicks, \& Bryan, 1999). In the case of inhibition of completed tasks, however, cognitive accessibility after success will drop below baseline. An inhibition or blocking of task-related information after success thus leads to a reduction in the strength of interference effects of the success-related words. Therefore, stronger interference effects for failure-related words relative to success-related words, as observed in the first experiment, can be attributed to a perseverance after failure, an inhibition after success, or a mixture of both.

Apparently, a comparison of failure and success conditions alone is not sufficient to decide which of these possible interpretations is correct. A similar interpretational problem also applies to most, if not all, of the previous studies in which the perseverance hypothesis was investigated. To disentangle effects of perseveration after failure versus inhibition after success, a second experiment was conducted in which interference effects of failureand success-related words were compared against a neutral baseline.

\section{EXPERIMENT 2}

In the second experiment, the same experimental arrangement was used as in the first experiment. The participants first had to work on a series of difficult synonym selection problems and received positive and negative feedback that was independent of their actual performance. In some interspersed synonym problems, however, the correct solution was already specified, and the participants were asked to indicate which of the remaining response alternatives would have dissuaded them most from finding the correct solution. No feedback was given for these problems. These interspersed synonym problems provided a neutral baseline against which the success and failure conditions were tested. Choosing the personally second-best response alternative did not specify a goal that could be reached or missed. On the other hand, the participants were required to process the task information in the same way as in those trials in which they had to identify the correct so- 
lution. A comparison of the success and failure conditions with the neutral baseline thus reflected pure effects of feedback and was not confounded with differences in the processing of the task-relevant information. In addition, for each participant, one set of synonym selection problems was not presented during the experiment. These synonym problems provided a second baseline condition that was introduced as a comparison against which the effects of the neutral processing condition were tested.

Automatic vigilance for the failure, success, neutral, and not-presented problems was measured in the second part of the experiment. The target words of the synonym problems were presented as distractor stimuli in a combined naming and tone detection task. Interference effects for these words in the combined task served as a measure of automatic vigilance. The perseverance-after-failure hypothesis predicts stronger interference effects for words from the negative feedback tasks, as compared with words from the no-feedback condition. The inhibitionafter-success hypothesis predicts weaker interference effects for words from the positive feedback tasks, as compared with the no-feedback condition. As in the previous experiment, stronger interference effects of the distractors were expected for the secondary task.

\section{Method}

Participants. Forty-eight University of Trier undergraduates (36 women and 12 men) volunteered in exchange for partial credit toward course requirements. None of them had participated in the first experiment. The experiment was conducted in individual sessions of approximately 30 -min duration.

Materials. The materials were the same as those in the first experiment, except for the following changes. Four additional synonym problems were selected as experimental problems. The resulting 12 experimental problems were split into four sets of 3 problems that were of comparable difficulty.

For the combined naming and tone detection task, 110 neutral word pairs were generated, the first 10 pairs of which served as practice trials. A tone was presented in 2 of the practice trials. From the remaining 100 pairs, 20 pairs were selected for tone presentation. In 12 of these trials, the target words of the experimental synonym problems were presented as distractor stimuli. The remaining 8 trials served as filler trials and contained distractor words that were unrelated to the synonym selection problems of the first phase.

Design. The conditions of the feedback factor (negative, positive, no feedback, or not presented) were manipulated within subjects for the experimental problems. Assignment of the four sets of experimental problems to the feedback conditions was counterbalanced across participants according to a Latin square. Position and sequence effects of feedback conditions during the experimental trials were counterbalanced across participants. Because the naming and tone detection task was presented twice (see the Procedure section), block (first vs. second presentation) was introduced as an additional factor.

Procedure. Procedural details were identical to those in the first experiment, with the following exceptions. (1) A total of nine experimental synonym problems was presented: three problems with predetermined positive feedback, three problems with predetermined negative feedback, and three problems without feedback, in which the correct solution was already prespecif ied and the participants had to select the second-best response alternative. The participants were told that the computer would make use of their answers to these tasks for selecting the following tasks. One set of three additional synonym problems was not presented to each par- ticipant. (2) The 100 trials of the combined naming and tone detection task were presented twice in two successive blocks. This should enhance the reliability of the interference measure by increasing the number of trials entering into the response time measures.

\section{Results}

Tone detection latencies. Reaction times in the secondary tone detection task were the primary dependent variable in the present experiment. Trials in which no response was registered until the beginning of the next trial ( $0.3 \%$ of all the trials) and outlier values (3.4\% of all the responses; see note 1 ) were not considered in the analyses. For each participant, mean reaction times were computed separately for the first and the second blocks for trials containing distractor stimuli referring to the four sets of experimental synonym problems. A 4 (distractor type) $\times 2$ (block) multivariate analysis of variance (MANOVA) revealed significant main effects for distractor type $\left[F(3,45)=3.90, M S_{\mathrm{e}}=1,757.24, p<.05\right]$ and block $\left[F(1,47)=59.28, M S_{\mathrm{e}}=2,600.70, p<.001\right]$. The interaction was nonsignificant $[F(3,45)=1.51$, $\left.M S_{\mathrm{e}}=1,501.01\right]$. Mean response latencies in the tone detection task are shown in Table 1 (latencies were averaged across blocks because the interaction of distractor type and block was nonsignificant).

Planned contrasts were conducted to test the perseverance hypothesis and the inhibition-after-success hypothesis and to analyze the effects of mere processing without feedback on automatic vigilance. In comparison with the trials with distractors from the no-feedback problems (baseline condition), tone detection latencies were $11 \mathrm{msec}$ longer for the trials containing the target words of the failure problems [by subjects, $t(47)=2.08$, $p<.05, d=.30$; by items, $t(11)=2.24, p<.05, d=.65$ ] Response times were $9 \mathrm{msec}$ shorter than in the baseline condition for the trials in which the target words of the success problems were presented [by subjects, $t(47)=$ $-1.73, p<.05$, one-tailed, $d=.25$; by items, $t(11)=$ $1.82, p<.05$, one-tailed, $d=.53]$. Reaction times did not differ significantly for the trials with distractor words from the no-feedback and the not-presented problems $(t<1)$.

Naming latencies. An analogous analysis of the naming latencies revealed only a significant main effect of block $\left[F(1,43)=7.35, M S_{\mathrm{e}}=2,839.46, p<.01\right]$, indicating faster responses in the second block (first block, $M=$ $595 \mathrm{msec}$; second block, $M=580 \mathrm{msec}$ ). The main effect of distractor type (failure, $M=588 \mathrm{msec}$; success, $M=$ $586 \mathrm{msec}$; no feedback, $M=585 \mathrm{msec}$; not presented, $M=591 \mathrm{msec})$ and the interaction of block and distractor type were nonsignificant (both $F \mathrm{~s}<1$ ). The lower degrees of freedom in this analysis are due to missing values, for some participants, that were caused by external noise, which interfered with the voice key mechanism.

\section{Discussion}

The results of the second experiment replicated the findings of the first one. The target words of failure problems produced stronger interference effects in a subsequent 
reaction time task than did the words relating to success problems. Partitioning this difference into a perseveranceafter-failure component and an inhibition-after-success component yielded two effects of comparable magnitude. The results thus supported both hypotheses.

Selecting the second-best response alternative when the correct solution was already specified did not alter the strength of interference effects, as compared with a second baseline condition in which the synonym problems were not presented at all. A mere processing of the content of a synonym problem without receiving feedback did not lead to a persistent increase (or inhibition) of automatic vigilance for task-related information.

In the previous experiment, perseverance and inhibition effects of failure- and success-related distractor words appeared only in the secondary tone detection task. No indication of these effects was present in the primary task. It was argued earlier that this asymmetry can be explained by the assumption that attentional capture by task-irrelevant distractors primarily affects executive control processes involved in switching between the tasks and, therefore, influences the responses that are executed second. So far, however, this argument has been merely hypothetical. Experiment 3 was conducted in order to test a direct implication of this assumption. According to the previous reasoning, distractor interference effects should affect the primary task, rather than the secondary task, when responses of the secondary task are executed first.

\section{EXPERIMENT 3}

An experimental arrangement was used similar to that in the second experiment. The participants first had to work on a series of difficult synonym selection problems and received positive or negative feedback that was independent of their actual performance. In a baseline condition, the correct solution was already specified, and no feedback was given for these problems. The additional baseline condition of not-presented problems did not differ from the neutral baseline condition in the second experiment and was, therefore, omitted.

Automatic vigilance for the failure, success, and neutral problems was measured in the second part of the experiment. As in the previous experiments, the target words of the synonym problems were presented as distractor stimuli in a combined task. Interference effects for these words in the combined task served as a measure of automatic vigilance. The nature of the combined task, however, was changed so that responses for the secondary task were now given before the responses for the primary task were executed. To achieve this sequence of responding, a word categorization task was used as the primary task, instead of the word-reading task of the preceding experiments. This task consisted in classifying German nouns according to their grammatical gender by pressing a left or a right response key (many German nouns have a masculine or feminine gender; e.g., Tisch [table] is masculine, Tasse [cup] is feminine). The secondary tone detection task consisted in simply saying the word Ton (tone) whenever a tone was emitted in one of the trials. A pilot study had revealed that with this combination, tone detection responses are given before categorization responses are executed. Nevertheless, the grammatical categorization task was still the primary task, because it had to be executed in each trial of the combined task, whereas the tone was presented in only one out of five trials.

The aim of the third experiment was to replicate the findings of Experiment 2: Words from the negative feedback synonym problems should produce stronger interference effects in the combined categorization and tone detection task than do words in the no-feedback condition (perseverance after failure), whereas words from the positive feedback problems should interfere less, as compared with the no-feedback condition (inhibition after success). This time, however, the pattern of interference effects was predicted to affect the primary task (grammatical categorization), because responses in this task were executed after the responses in the secondary tone detection task.

\section{Method}

Participants. Forty-two University of Trier undergraduates (33 women and 9 men) volunteered in exchange for partial credit toward course requirements. None of them had participated in the previous experiments. The experiment was conducted in individual sessions of approximately 30-min duration.

Materials. The materials were the same as those in the second experiment, except for the following changes. Owing to the omission of the second baseline condition, only nine synonym selection problems received manipulated feedback. The nine problems were split into three sets of three problems.

For the combined categorization and tone detection task, 120 target / distractor pairs were generated, the first 20 pairs of which served as practice trials. A tone was presented in four of the practice trials. From the remaining 100 pairs, 18 pairs were selected for tone presentation. In all of the tone trials, the target word of one of the nine experimental synonym problems was presented as a distractor. Each of the target words of the synonym selection problems was presented twice in the combined task, once with a masculine target and once with a feminine target.

Design. The conditions of the feedback factor (negative, positive, or no feedback) were manipulated within subjects for the experimental problems. Assignment of the three sets of experimental problems to the feedback conditions was counterbalanced across participants according to a Latin square. Position and sequence effects of feedback conditions during the experimental trials were counterbalanced across participants.

Procedure. The procedural details were identical to those in the second experiment, except for the following changes. (1) Responses in the categorization task were given by pressing one of two keys on a computer keyboard. (2) Responses in the tone detection task were given by saying the word Ton (tone). Response latencies were registered by a voice key apparatus realized by means of a microphone connected to a SoundBlaster audio card. (3) The word stimuli remained on the screen until a categorization response was registered and were not masked after presentation. The next trial was initiated $500 \mathrm{msec}$ after the categorization response. In the case of a tone trial, the following trial was initiated $500 \mathrm{msec}$ after the last of the two responses was registered. (4) The 100 trials of the combined 
categorization and tone detection task were presented twice in two successive blocks. The response assignment of the categorization task was switched between blocks, to prevent an automatization of the task.

\section{Results}

Categorization latencies. Reaction times in the categorization task were the primary dependent variable in the present experiment. Trials in which an error occurred (6.1\% of all the trials) and outlier values $(2.9 \%$ of all the responses; see note 1) were not considered in the analyses. For each participant, mean response times were computed separately for the first and the second blocks for trials containing distractor stimuli referring to the four sets of experimental synonym problems. A 3 (distractor type) $\times 2$ (block) MANOVA revealed significant main effects for distractor type $\left[F(2,40)=9.65, M S_{\mathrm{e}}=\right.$ $24,943.26, p<.001]$ and block $\left[F(1,41)=10.89, M S_{\mathrm{e}}=\right.$ $116,550.77, p<.01]$. The interaction was nonsignificant $\left[F(2,40)=1.46, M S_{\mathrm{e}}=43,584.34\right]$. Mean categorization latencies are shown in Table 1 (latencies were averaged across blocks, because the interaction of distractor type and block was nonsignificant).

Planned contrasts were conducted to test the perseverance hypothesis and the inhibition-after-success hypothesis and to analyze effects of mere processing without feedback on automatic vigilance. For these analyses, categorization latencies were averaged across blocks, because the interaction of block and distractor type was nonsignificant. In comparison with trials with distractors from the no-feedback problems (baseline condition), categorization latencies were $42 \mathrm{msec}$ longer for trials containing the target words of the failure problems [by subjects, $t(41)=1.79, p<.05$, one-tailed, $d=.28$; by items, $t(8)=1.28, p>.10, d=.43]$. Response times were $65 \mathrm{msec}$ shorter than in the baseline condition for trials in which the target words of the success problems were presented [by subjects, $t(41)=2.42, p<.05, d=.37$; by items, $t(8)=1.97, p<.05$, one-tailed, $d=.66]$.

Tone detection latencies. An analogous analysis of the tone detection latencies revealed only a significant main effect of block $\left[F(1,41)=6.32, M S_{\mathrm{e}}=52,373.37\right.$, $p<.05$ ], indicating slower responses in the second block (first block, $M=689 \mathrm{msec}$; second block, $M=762 \mathrm{msec}$ ). The main effect of distractor type (failure, $M=684 \mathrm{msec}$; success, $M=758 \mathrm{msec}$; no feedback, $M=734 \mathrm{msec}$ ) was nonsignificant $\left[F(1,41)=2.50, M S_{\mathrm{e}}=48,104.85\right]$, and the interaction of distractor type and block was also nonsignificant $(F<1)$.

\section{Discussion}

The results of the third experiment replicated the findings of the second one. Words relating to failure problems produced stronger interference effects in a subsequent reaction time task than did the words relating to success problems. Partitioning this difference into a perseveranceafter-failure component and an inhibition-after-success component yielded two effects of comparable magnitude. The perseverance and inhibition effects (difference scores) were much larger, in terms of milliseconds, than those in the previous experiment. This was possibly due to the increased difficulty of the combined task in the present task, resulting in much higher response latencies, which also inflated differences.

In contrast to the previous experiment, however, perseverance and inhibition effects of failure- and successrelated distractor words were found only in the primary grammatical categorization task, and not in the secondary tone detection task. This pattern of findings corresponds to the prediction that distractor interference primarily affects processes of task switching. According to this account, a fast or slow switching between the tasks will have an effect on responding only for the task that is executed second. Therefore, differential effects of distractor interference on task switching should have an influence mainly in the grammatical categorization task, because responses in the tone detection task were given before the responses in the grammatical categorization task were executed.

\section{GENERAL DISCUSSION}

Adoption of a goal or a task is typically accompanied by a corresponding attentional set. According to Lewin (1926, 1935), this automatic vigilance mechanism should persist even after the goal or the task has definitely been failed, thereby perpetuating an increased sensitivity for information that is no longer relevant for the regulation of behavior (perseverance hypothesis). Three experiments were conducted to test this hypothesis. The major aim of the present experiments was to avoid a biasing of results by strategic processes. For this purpose, an indirect measure of automatic vigilance was employed. Words relating to previous success and failure tasks were presented as distractor stimuli in a subsequent reaction time task. Interference effects for these words were used to measure the persistence of automatic vigilance for taskrelated information. All the experiments revealed significant effects of feedback on the persistence of automatic vigilance: Words relating to synonym problems that had received negative feedback produced stronger interference effects in the reaction time task than did words relating to problems that had received positive feedback.

The second and third experiments also included a neutral baseline in order to separate effects of failure and success feedback on automatic vigilance. Failure feedback was associated with increased interference effects in the reaction time task. This finding specifically supports the perseverance hypothesis. Apparently, commitment to a goal or a task induces a persistent increase in automatic vigilance that is highly resistant to negative feedback and persists despite situational and behavioral changes. In the present experiments, task-related information was irrelevant after a response alternative had been selected and feedback had been given. Each synonym selection problem was presented only once during the experiment, and the participants had no reason to ex- 
pect that they would encounter the synonym problems again. Stability of task-related attentional sets in the face of irreversible failure thus does not result from rational deliberation or adaptive persistence and tenacity in pursuing goals but, rather, reflects a general tendency toward perseveration. Perseverance at the level of concrete synonym selection problems on which participants had (ostensibly) failed, however, might be fueled by a persisting commitment to the superordinate goal of demonstrating their intelligence. ${ }^{3}$ Evidence for such a link between higher and lower order goals comes from studies showing that ruminative thought after failure in a specific task can be reduced by providing self-affirmative feedback relating to the superordinate goal for which the task is relevant (Brunstein \& Gollwitzer, 1996; Koole, Smeets, van Knippenberg, \& Dijksterhuis, 1999). Such a persistence at the level of the superordinate goal might be adaptive even in the face of failure at the subordinate level, because it disposes the organism to search for alternative means to attain the superordinate goal (Kuhl, 1987).

Staying committed to the superordinate goal, however, does not explain why people should remain committed to the concrete tasks that have become definitely unattainable, nor does it render these specific perseverance effects rational or adaptive. To the contrary, maintaining an active set relating to a concrete task at which one has irreversibly failed might delay processes of disengagementand reorientation toward alternative ways to reach superordinate goals (Brandtstädter \& Rothermund, 2002a, 2002b; Wrosch, Scheier, Carver, \& Schulz, 2003). An automaticand perhaps even counterintentional-persistence of goalrelated attentional sets after failure might also provide a key to the understanding of dysfunctional phenomena, such as rumination (Martin \& Tesser, 1989), or behavioral perseverance after failure (as demonstrated, e.g., in the inability to ignore sunk costs [Arkes \& Ayton, 1999] and the tendency of escalating commitment to a losing course of action [Staw, 1976, 1997]).

A second important finding was that success feedback reduced interference effects, as compared with the neutral baseline. In accordance with recent findings by Marsh et al. (1998; Marsh et al., 1999), the latter effect indicates a suppression of task-related information after the successful completion of a task. An inhibitory mechanism that terminates previously established goal- or task-related attentional sets after having reached a success criterion functions as a useful counterpart to the vigilance mechanism. Such an inhibitory mechanism provides an efficient means to eradicate previous attentional control settings and to prevent a persistence of automatic vigilance for information relating to already completed goals and tasks that are no longer relevant for the regulation of behavior.

Across experiments, effects were somewhat stronger (in terms of $d$ ) in the by-items analyses than in the bysubjects analyses. This suggests that effects of positive and negative feedback vary systematically between individuals, because interindividual differences in the strength or direction of the feedback effect contribute to the error variance in the by-subjects analyses, but not in the byitems analyses (Clark, 1973). Analyzing moderating effects of dispositional variables on the relation between feedback and attention may, therefore, be a promising line for future research (Brandtstädter, Wentura, \& Rothermund, 1999; Goschke \& Kuhl, 1993).

On a methodological level, it has been argued in the introduction that an analysis of interference effects provides an unbiased measure of automatic vigilance. Application of such an indirect measure is of prime importance when motivationally relevant information is used-that is, when the stimulus materials are connected to an "ego task" that might become superimposed onto the actual task (Greenwald, 1982). But of course, the use of interference measures is not limited to these specific circumstances. Interference effects can generally be used as an implicit measure of automatic cognitive accessibility that is not contaminated with strategic processes (Jacoby, 1991). This kind of measure might provide an interesting alternative to other implicit measures of memory and might be applicable even when process dissociation procedures are difficult to implement.

The findings of the present experiments also revealed an interesting dissociation in the pattern of distractor interference effects that emerged for the different tasks of the dual-task arrangements. Across experiments, interference effects were much stronger for the task that was executed last, regardless of whether this task constituted the primary task or the secondary task of the combined task. On the other hand, no consistent pattern of interference effects emerged for those tasks that were executed first. This asymmetry in distractor interference effects is compatible with the assumption that distractor interference primarily affects executive control processes involved in switching between the different tasks of the dual-task arrangement. The use of combined tasks is, therefore, highly recommended when indirect and subtle effects of distractor interference are analyzed.

\section{REFERENCES}

Anderson, J. R. (1983). The architecture of cognition. Cambridge, MA: Harvard University Press.

Arkes, H. R. \& Ayton, P. (1999). The sunk cost and Concorde effects: Are humans less rational than lower animals? Psychological Bulletin, 125, 591-600.

BARGH, J. A. (1982). Attention and automaticity in the processing of self-relevant information. Journal of Personality \& Social Psychology, 43, 425-436.

BARGH, J. A. (1989). Conditional automaticity: Varieties of automatic influence in social perception and cognition. In J. S. Uleman \& J. A. Bargh (Eds.), Unintended thought (pp. 3-51). New York: Guilford. BeckmAnN, J. (1996). Self-presentation and the Zeigarnik effect. In T. Gjesme \& R. Nygard (Eds.), Advances in motivation (pp. 35-45). Oslo: Scandinavian University Press.

BRAndtst ÄDter, J., \& Renner, G. (1992). Coping with discrepancies between aspirations and achievements in adult development: A dualprocess model. In L. Montada, S.-H. Filipp, \& M. J. Lerner (Eds.), Life crises and experiences of loss in adulthood (pp. 301-319). Hillsdale, NJ: Erlbaum.

BRANDTST ÄDTER, J., \& Rothermund, K. (2002a). Intentional selfdevelopment: Exploring the interfaces between development, intentionality, and the self. In L. J. Crockett (Ed.), Agency, motivation, and 
the life course (Nebraska Symposium on Motivation, Vol. 48, pp. 31 75). Lincoln: University of Nebraska Press.

BRAndtstädter, J., \& Rothermund, K. (2002b). The life-course dynamics of goal pursuit and goal adjustment: A two-process framework. Developmental Review, 22, 117-150.

BRAndtstäDter, J., Wentura, D., \& Rothermund, K. (1999). Intentional self-development through adulthood and later life: Tenacious pursuit and flexible adjustment of goals. In J. Brandtstädter \& R. M. Lerner (Eds.), Action and self-development: Theory and research through the life-span (pp. 373-400). Thousand Oaks, CA: Sage.

Brunstein, J. C., \& Gollwitzer, P. M. (1996). Effects of failure on subsequent performance: The importance of self-defining goals. Journal of Personality \& Social Psychology, 70, 395-407.

ButTerfield, E. C. (1964). The interruption of tasks: Methodological, factual, and theoretical issues. Psychological Bulletin, 62, 309-322.

Caron, A. J., \& Wallach, M. A. (1957). Recall of interrupted tasks under stress: A phenomenon of memory or of learning? Journal of Abnormal \& Social Psychology, 55, 372-381.

Clark, H. H. (1973). The language-as-fixed-effect fallacy: A critique of language statistics in psychological research. Journal of Verbal Learning \& Verbal Behavior, 12, 335-359.

Derryberry, D., \& Tucker, D. M. (1994). Motivating the focus of attention. In P. M. Niedenthal \& S. Kitayama (Eds.), The heart's eye (pp. 167-196). San Diego: Academic Press.

ENGLE, R. W. (2002). Working memory capacity as executive attention. Current Directions in Psychological Science, 11, 19-23.

ERIKSEN, C. W. (1958). Unconscious processes. In M. R. Jones (Ed.), Nebraska symposium on motivation (Vol. 6, pp. 169-227). Lincoln: University of Nebraska Press.

Goschke, T., \& KuHL, J. (1993). Representation of intentions: Persisting activation in memory. Journal of Experimental Psychology: Learning, Memory, \& Cognition, 19, 1211-1226.

Goschke, T., \& KuHL, J. (1996). Remembering what to do: Explicit and implicit memory for intentions. In M. Brandimonte, G. O. Einstein, \& M. A. McDaniel (Eds.), Prospective memory: Theory and applications (pp. 53-91). Hillsdale, NJ: Erlbaum.

GREENWALd, A. G. (1982). Ego task analysis: An integration of research on ego-involvement and self-awareness. In A. H. Hastorf \& A. Isen (Eds.), Cognitive social psychology (pp. 109-147). New York: Elsevier.

HoLmES, D. S. (1990). The evidence of repression: An examination of sixty years of research. In J. L. Singer (Ed.), Repression and dissociation (pp. 85-102). Chicago: University of Chicago Press.

Houghton, G., \& Tipper, S. P. (1994). A model of inhibitory mechanisms in selective attention. In D. Dagenbach \& T. H. Carr (Eds.), Inhibitory processes in attention, memory, and language (pp. 53-112). San Diego: Academic Press.

JACOBY, L. L. (1991). A process dissociation framework: Separating automatic from intentional uses of memory. Journal of Memory \& Language, 30, 513-541.

JÄGER, A. O., \& ALTHOFF, K. (1983).Der WILDE-Intelligenztest(WIT): Ein Strukturdiagnostikum. Göttingen: Hogrefe.

KLINGER, E. (1975). Consequences of commitment to and disengagement from incentives. Psychological Review, 82, 1-25.

KLINGER, E. (1996). The contents of thoughts: Interference as the downside of adaptive normal mechanisms in thought flow. In I. G. Sarason, G. R. Pierce, \& B. R. Sarason (Eds.), Cognitive interference: Theories, methods, and findings (pp. 3-23). Mahwah, NJ: Erlbaum.

Koole, S. L., Smeets, K., van Knippenberg, A., \& Dijksterhuis, A. (1999). The cessation of rumination through self-affirmation. Journal of Personality \& Social Psychology, 77, 111-125.

KuHL, J. (1987). Action control: The maintenance of motivational states. In F. Halisch \& J. Kuhl (Eds.), Motivation, intention, and volition (pp. 279-291). Berlin: Springer-Verlag.

KuHL, J., \& HeLle, P. (1986). Motivational and volitional determinants of depression: The degenerated-intention hypothesis. Journal of Abnormal Psychology, 95, 247-251.

LewIN, K. (1926). Vorsatz, Wille und Bedürfnis. Psychologische Forschung, 7, 330-385.

LEWIN, K. (1935). A dynamic theory of personality. New York: McGrawHill.

Marrow, A. J. (1938). Goal tensions and recall: II. Journal of General Psychology, 19, 37-64.
Marsh, R. L., Hicks, J. L., \& BinK, M. L. (1998). Activation of completed, uncompleted, and partially completed intentions. Journal of Experimental Psychology: Learning, Memory, \& Cognition, 24, 350-361.

Marsh, R. L., Hicks, J. L., \& BRYAN, E. S. (1999). The activation of unrelated and canceled intentions. Memory \& Cognition, 27, 320-327.

Martin, L. L., \& Tesser, A. (1989). Toward a motivational and structural theory of ruminative thought. In J. S. Uleman \& J. A. Bargh (Eds.), Unintended thought (pp. 306-326). New York: Guilford.

Martin, L. L., Tesser, A., \& McIntosh, W. D. (1993). Wanting but not having: The effects of unattained goals on thoughts and feelings. In D. M. Wegner \& J. W. Pennebaker (Eds.), Handbook of mental control (pp. 552-572). Englewood Cliffs, NJ: Prentice-Hall.

Mayr, U., \& KLIEgL, R. (2000). Task-set switching and long-term memory retrieval. Journal of Experimental Psychology: Learning, Memory, \& Cognition, 26, 1124-1140.

McCABE, S. B., \& GotLib, I. H. (1993). Attentional processing in clinically depressed subjects: A longitudinal investigation. Cognitive Therapy \& Research, 17, 359-377.

Moskowitz, G. B. (2002). Preconscious effects of temporary goals on attention. Journal of Experimental Social Psychology, 38, 397-404.

PASHLER, H. E. (1998). The psychology of attention. Cambridge, MA: MIT Press.

Posner, M. I., \& Boies, S. J. (1971). Components of attention. Psychological Review, 79, 391-408.

Postman, L., \& Solomon, R. L. (1949). Perceptual sensitivity to completed and incompleted tasks. Journal of Personality, 18, 347-357.

RiemanN, B. C., \& McNally, R. J. (1995). Cognitive processing of personally relevant information. Cognition \& Emotion, 9, 325-340.

Rothermund, K., Wentura, D., \& BaK, P. (2001). Automatic attention to stimuli signalling chances and dangers: Moderating effects of positive and negative goal and action contexts. Cognition \& Emotion, 15, 231-248.

Rubinstein, J. S., Meyer, D. E., \& Evans, J. E. (2001). Executive control of cognitive processes in task switching. Journal of Experimental Psychology: Human Perception \& Performance, 27, 763-797.

Russo, R, \& PARKIn, A. J. (1993). Age differences in implicit memory: More apparent than real. Memory \& Cognition, 21, 73-80.

STAw, B. M. (1976). Knee-deep in the Big Muddy: A study of escalating commitment to a chosen course of action. Organizational Behavior \& Human Decision Processes, 16, 27-44.

STAW, B. M. (1997). The escalation of commitment: An update and appraisal. In Z. Shapira (Ed.), Organizationaldecision making (pp. 191215). Cambridge: Cambridge University Press.

Tukey, J. W. (1977). Exploratory data analysis. Reading, MA: AddisonWesley.

van Bergen, A. (1968). Task interruption. Amsterdam: Elsevier, NorthHolland.

Wrosch, C., Scheier, M. F., Carver, C. S., \& Schulz, R. (2003). The importance of goal disengagement in adaptive self-regulation: When giving up is beneficial. Self \& Identity, 2, 1-20.

ZEIGARNIK, B. (1927). Das Behalten erledigter und unerledigter Handlungen. Psychologische Forschung, 9, 1-85.

\section{NOTES}

1. Reaction times that were more than two interquartile ranges above the median of the distribution of reaction times were considered outlier values (Tukey, 1977). Outlier criteria were determined on the basis of only those trials in which responses for both the primary and the secondary tasks had to be executed. Trimming the data of the task that is executed second effectively trimmed the data of the task for which responses are given first.

2. Although average response latencies were slightly longer for the reading task than for the tone detection task, reading responses were nevertheless initiated first because measurement of tone detection latencies started with the onset of the tone, which appeared $300 \mathrm{msec}$ after the onset of the words.

3. This possibility was suggested by Leonard Martin.

(Manuscript received May 21, 2002; revision accepted for publication January 28, 2003.) 